\title{
Remodeling of Neuromuscular Junctions in Adult Mouse Soleus
}

\author{
Donald J. Wigston \\ Department of Physiology, Emory University School of Medicine, Atlanta, Georgia 30322
}

\begin{abstract}
The stability of neuromuscular junctions (NMJs) was studied in soleus muscles of adult mice by labeling acetylcholine receptors in vivo with rhodamine $\alpha$-bungarotoxin. Identified NMJs were examined in living animals by low-light-level fluorescence microscopy on 2 or 3 occasions separated by up to 6 months. Many NMJs appeared identical each time they were viewed except for overall enlargement probably related to growth of the animal. Forty-four percent of NMJs, however, changed their shape over 6 months; these changes consisted mostly of small deletions or additions to part of the initial configuration. NMJs in adult soleus appeared to be less malleable than suggested by earlier studies but more plastic than NMJs in another muscle, the mouse sternomastoid, in which virtually no remodeling was observed using similar methods to the present study (Lichtman et al., 1987a). Thus, the degree of remodeling at NMJs may vary among different muscles, perhaps depending on their pattern of use.
\end{abstract}

It has often been suggested that synaptic connections are continually rejuvenated by remodeling. $\Lambda$ capacity for anatomical remodeling of synaptic connections might underlie long-term changes in synaptic connections such as alterations in synaptic efficacy during learning (see, for example, Bailey and Chen, $1983,1988)$ or in response to the pattern of synaptic usage (Lnenicka et al., 1986). Indeed, evidence in favor of the remodeling of synapses has been described in the normal mammalian CNS (Sotelo and Palay, 1971). However, the evidence for anatomical remodeling of CNS synapses is necessarily indirect, since it has not so far been possible to examine individual central synapses more than once in the lifetime of an animal.

The accessibility of the PNS, however, allows the important issue of whether synaptic connections are stable or dynamic to be addressed directly. The advent of sensitive video cameras and fluorescent probes that delineate certain aspects of synaptic morphology means that it is now possible to study peripheral synaptic connections visually in living animals (Purves and Voyvodic, 1987). For example, fluorescent probes are available that highlight presynaptic terminals (Yoshikami and Okun, 1984; Magrassi et al., 1987; Purves et al., 1987; Robbins and Polak, 1987), postsynaptic dendrites (Purves et al., 1986), postsynaptic receptors (Lichtman et al., 1987b), or synapse-specific extracellular matrix (Sanes and Cheney, 1982; Kelly et al., 1985; Ko,

Received May 17, 1988; revised July 15, 1988; accepted July 18, 1988

I thank J. Huffaker and P. Shuler for assistance, and R. Balice-Gordon, V. Boss, L. Crews, S. Donahue, A. English, A. Herrera, J. Lichtman, and D. Purves for helpful suggestions. This work was supported by grants from Emory University, $\mathrm{MDA}$, and $\mathrm{NIH}$

Correspondence should be addressed to D. J. Wigston at the above address.

Copyright (C) 1989 Society for Neuroscience $0270-6474 / 89 / 020639-09 \$ 02.00 / 0$
1987; Scott et al., 1988). If low levels of illumination are used that do not damage the structure being studied, there is, in principle, no limit to the number of times a particular set of synapses can be examined during the life of an animal. This approach has been used successfully to study superficial regions of the nervous system, such as pre- or postsynaptic elements in autonomic ganglia (Purves et al., 1986, 1987), or the arrangement of motor nerve terminals in muscle (Lichtman et al., 1987a) over several months.

Synaptic connections in vertebrate skeletal muscle are particularly suitable for in vivo imaging. Using this approach, Herrera and Banner (1987) have observed substantial remodeling of the majority of nerve terminals in frog muscle, as had been suggested by earlier histological and ultrastructural studies (Wernig et al., 1980). On the other hand, very little remodeling of neuromuscular junctions (NMJs) $(<1 \%)$ was observed in the mouse sternomastoid muscle, studied in vivo over time (Lichtman et al., 1987a). The apparent lack of remodeling of NMJs in sternomastoid is at odds with the conclusions of earlier studies of mammalian NMJs using histological and ultrastructural techniques (Barker and Ip, 1966; Tuffery, 1971; Cardasis and Padykula, 1981; Wernig et al., 1984; Rotshenker and Tal, 1985), in which evidence consistent with the ongoing sprouting and retraction of nerve terminals in normal muscle was observed. The most detailed evidence for remodeling has come from rat or mouse soleus (Cardasis and Padykula, 1981; Wernig et al., 1984). However, since the sternomastoid muscle studied by Lichtman et al. (1987a) is a fast-twitch muscle, whereas soleus is a slow-twitch muscle, the apparent difference between soleus and sternomastoid in the amount of NMJ remodeling might reflect a difference in the frequency of NMJ remodeling between slow- and fast-twitch muscles. Alternatively, the difference between the amount of remodeling observed at soleus and sternomastoid NMJs might be a consequence of different technical approaches, i.e., static versus dynamic observations. To help distinguish between these explanations, I followed the morphology of NMJs in mouse soleus muscles over time with lowlight-level fluorescence microscopy as has been done for the sternomastoid. My results indicate that a moderate degree of NMJ remodeling does occur in mouse soleus. Some of these results have been reported previously (Wigston, 1987).

\section{Materials and Methods}

This study mostly used male mice (C57B / $/ 6 \mathrm{~J}$ ), obtained from Jackson Labs or bred locally. For viewing NMJs, a $25-35$ gm mouse was anesthetized with chloral hydrate $(400 \mathrm{mg} / \mathrm{kg})$ and the lateral surface of its right hind leg shaved. The animal was then mounted on a moveable platform. A polyethylene tube was then inserted through the mouth into the trachea, and the skin overlying the right lateral gastrocnemius muscle (LG) was washed with antiseptic solution and incised. After gently pulling the LG posteriorly, the soleus was reflected to expose its anterior 

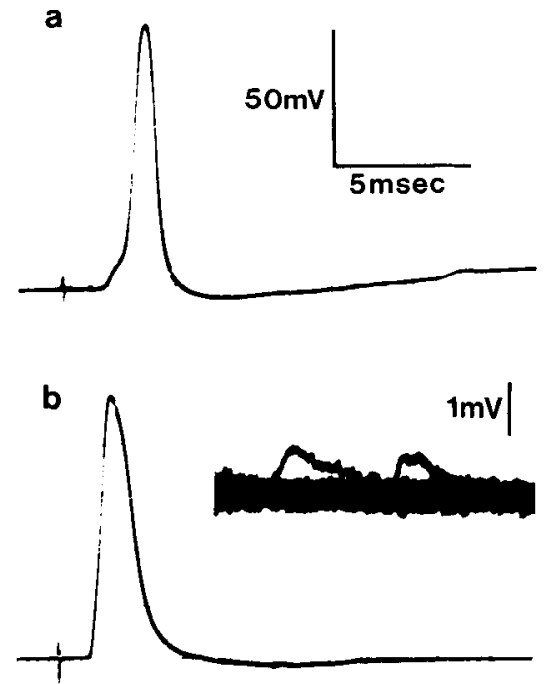

Figure 1. Intracellular recordings from superficial soleus muscle fibers in vitro. $a$, An action potential evoked by nerve stimulation immediately after staining ACh receptors with rhodamine-labeled $\alpha$-BTX for $1 \mathrm{hr}$. The small depolarization towards the right of the trace may be a miniature endplate potential. $b$, Nerve-evoked action potential recorded 4 $\mathrm{d}$ after labeling AChRs with R- $\alpha \mathrm{BTX}$. Inset, Two minature endplate potentials recorded from the same fiber at higher gain (several sweeps superimposed; $1 \mathrm{mV}$ calibration bar applies only to inset). In both a and $b$, fluorescent labeling of NMJs was verificd immediatcly after staining and was still detectable just prior to making the recordings in $b$. Scale bar in $a$ also applies to action potential in $b$.

surface. Since fluorescent nerve terminal stains worked poorly in soleus (see Discussion), I applied tetramethylrhodamine isothiocyanate-conjugated $\alpha$-bungarotoxin (R- $\alpha \mathrm{BTX}$ ), $\sim 5 \mu \mathrm{g} / \mathrm{ml}$ in oxygenated Ringer's (Molecular Probes) to the anterior surface of soleus to label acetylcholine receptors (AChRs). The fluorescent toxin was replaced several times over 30-40 min, and the muscle rinsed with oxygenated Ringer's. The platform on which the animal was mounted was then attached to the stage of a compound microscope equipped for epifluorescence and the endotracheal tube connected to a respirator. Supplementary doses of chloral hydrate $(100 \mathrm{mg} / \mathrm{kg})$ were administered approximately every half-hour.

Labeled NMJs in soleus were epi-illuminaed with a $100 \mathrm{~W}$ mercury arc lamp, heavily attenuated, and viewed with a $50 \times$ water-immersion objective lens (N.A. $=1.0$ ). Wavelengths appropriate for the excitation and emission of rhodamine fluorescence were selected with standard filters (Leitz N2 filter set). The incident light beam passed through 2 heat filters and a continously variable neutral density filter and was controlled with a shutter. The illumination intensity was adjusted with the variable density filter to the minimum necessary to obtain acceptable images with an intensified video camera (SIT) operated at maximum gain and black level. At no time were NMJs viewed through the microscope eyepieces since this required much more intense illumination. The output of the SIT camera was led to a digital image processor (Hughes 694 or Universal Imaging Corporation Image-1 AT). Live images were recorded continuously on videotape, even when moving the microscope ficld to locate new NMJs. This helped in the relocation of NMJs at later viewing sessions. Video images of selected junctions were processed to reduce camera noise (16-32 frame averages), contrastenhanced, and stored on videotape and in digital form on magnetic or optical disks (Maxtor). After studying 5-12 NMJs, the wound was closed with $6-0$ sutures and the animal was returned to its cage.

After an interval of $7-181 \mathrm{~d}$, the viewing procedure was repeated as at first. At the final viewing, a considerably higher concentration of R- $\alpha$ BTX $(10 \mu \mathrm{g} / \mathrm{ml})$ was used since this shortened the time required for adequate labeling. NMJs were relocated using sketches of each junction and the video recordings as guides, and new images of the same NMJs were obtained. In most experiments, after the final viewing the animal was perfused through the heart with saline and the right soleus muscle was removed. Nerve terminals in the muscle were then stained with tetranitroblue tetrazolium (TNBT; Lctinsky, 1983; Jordan et al., 1988). Junctional cholinesterase (ChE) was revealed by the method of Karnovsky (1964). After staining, the fibers from the anterior surface of soleus were removed as a thin sheet and mounted on a slide with Aquamount and a coverslip. The NMJs that had been studied in vivo were then relocated, compared directly with video images of R- $\alpha$ BTX fluorescence, and drawn or photographed using a $100 \times$ objective (N.A $=1.32$ ). Video images of $\mathrm{R}-\alpha \mathrm{BTX}$-stained junctions were photographed from a monitor with a $35 \mathrm{~mm}$ camera using Kodak Technical Pan film and automatic exposure control. Measurements were made from photographs with a ruler or directly from video data using an image analysis program (Analytical Imaging Concepts). Only elements showing clear changes were measured. No attempt was made to quantify the overall size or equivalent nerve terminal area of individual NMJs.

To examine the physiological effects of the viewing proccdurc, I recorded from muscles whose NMJs had been labeled with R- $\alpha$ BTX and viewed in vivo. The soleus muscle on the right side was stained with $\mathrm{R}-\alpha \mathrm{BTX}$ as above, and individual NMJs were examined to verify staining. Either immediately, $24 \mathrm{hr}$, or $4 \mathrm{~d}$ later, the animals were reanesthetized and perfused through the heart with an oxygenated Ringer's solution. In each animal, the right soleus muscle was removed and pinned out in a Sylgard-lined dish. The dish was then mounted on the stage of a compound microscope and continuosly superfused with normal oxygenated Ringer's solution of the following composition (mM): $\mathrm{NaCl}$, $137 ; \mathrm{KCl}, 4 ; \mathrm{MgSO}_{4}, 1 ; \mathrm{CaCl}_{2}, 1.8 ; \mathrm{HEPES}, 5 ;$ glucose, 11 . Superficial muscle fibers were viewed at $200 \times$ magnification and impaled with $3 \mathrm{M}$ $\mathrm{KCl}$-filled glass microelectrodes under visual guidance. The cut soleus nerve was stimulated with a suction electrode $(0.5-5 \mathrm{~V}, 50 \mu \mathrm{sec}, 0.5$ $\mathrm{Hz}$ ), and stimulus-cvoked and spontancous potential changes were recorded and displayed on a storage oscilloscope.

\section{Results}

\section{Effect of staining with $R-\alpha B T X$ on neuromuscular transmission}

A concern with using R- $\alpha \mathrm{BTX}$ to label NMJs in vivo was that it might induce nerve sprouting through its paralyzing action on muscles (Holland and Brown, 1980). To reduce the likelihood of causing prolonged paralysis and thereby inducing nerve sprouting, I used the lowest concentration of R- $\alpha$ BTX with which I could obtain acceptable fluorescent labeling of NMJs within $30-40 \mathrm{~min}(\sim 5 \mu \mathrm{g} / \mathrm{ml})$, without having to employ strong illumination ( $<5 \%$ transmission). In control experiments, I examined the effect of the imaging procedure on synaptic transmission at soleus $\mathrm{NMJ}$, by intracellular recording after staining and viewing NMJs in the usual way. In normal Ringer's solution, stimulation of the soleus nerve evoked action potentials (APs) in 24 of 25 superficial muscle fibers penetrated in 3 soleus muscles 1-2 hr after applying R- $\alpha$ BTX. Similar results were obtained 24 hr ( 2 muscles; 16/20 fibers) or 4 d (3 muscles; 30/31 fibers) after toxin treatment (see Fig. 1). Synaptic potentials (1-12 mV) were detected in the few cases where APs were absent. Moreover, spontaneous miniature endplate potentials were recorded in most of the fibers penetrated at each time. At the concentrations and incubation times I generally employed, then, R- $\alpha$ BTX appeared to paralyze only a small fraction of muscle fibers. However, the safety factor was probably reduced at all NMJs exposed to $\mathrm{R}-\alpha \mathrm{BTX}$, suggesting that at normal in vivo firing frequencies transmission might fall below threshold for initiation of action potentials at more NMJs than found using low-frequency stimulation in vitro. In any case, the partial paralysis was probably transient, since even after complete blockage of neuromuscular transmission, virtually all muscle fibers regain their ability to fire an action potential in response to a single nerve stimulus within about $24 \mathrm{hr}$ (Rochel and Robbins, 1987). This rapid recovery can probably be accounted for by the normal rate of insertion of new AChRs into the myofiber plasma membrane. 

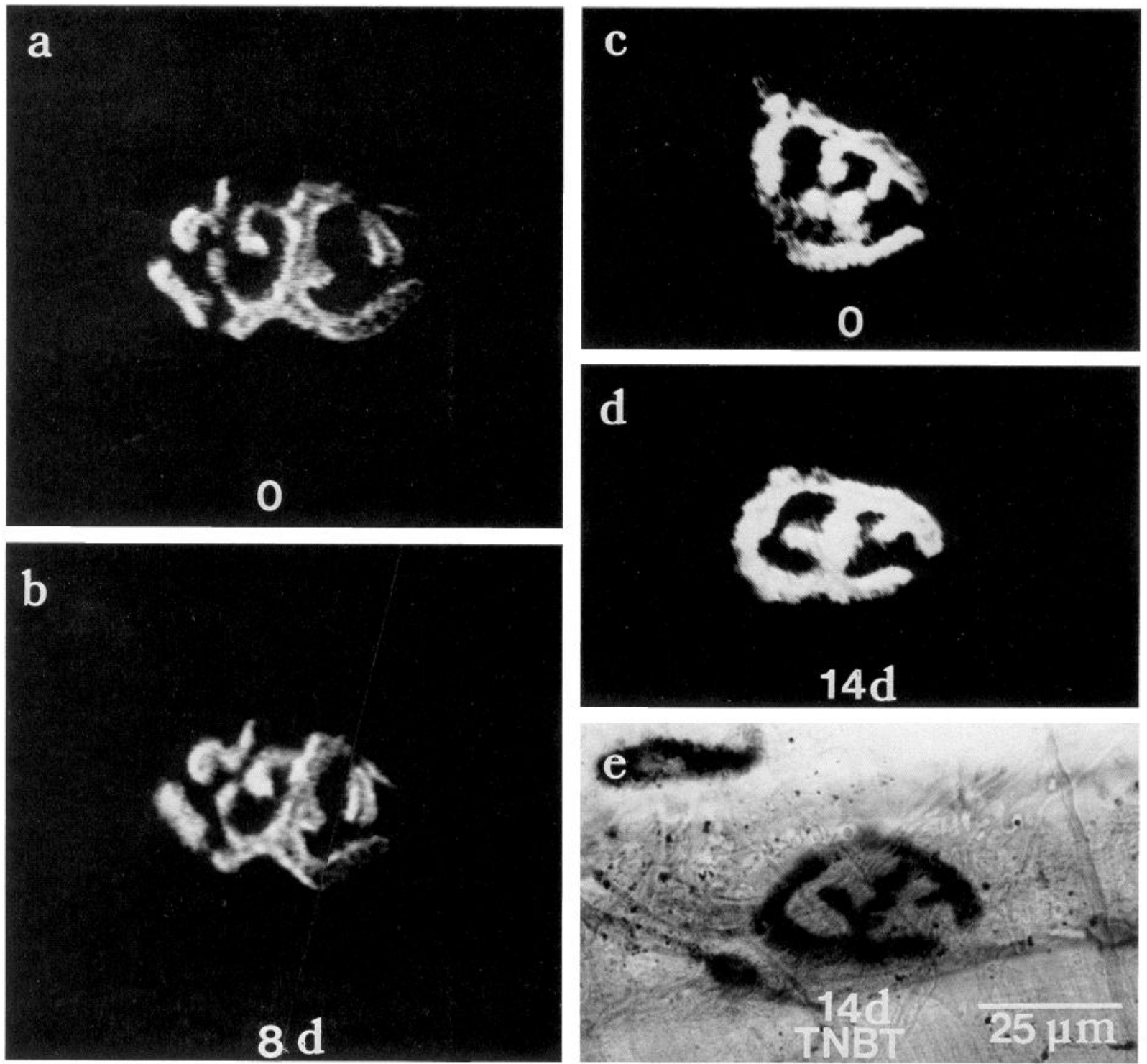

Figure 2. NMJs reexamined at relatively short times after the initial viewing. $a$ and $b$, Views of the same NMJ, labeled with R- $\alpha$ BTX and examined in vivo on 2 occasions separated by $8 \mathrm{~d}$. $c$ and $d$, Two views of another NMJ stained with R- $\alpha \mathrm{BTX}$ and studied in vivo on 2 occasions separated by $14 \mathrm{~d} . e$, Same NMJ as in $c$ and $d$, stained with TNBT to reveal the nerve terminal. In no NMJs reexamined at such short times after the initial viewing ( $<3$ weeks) were significant differences observed between the first and subsequent viewings except distortions as evident in $c-e$. The region at the bottom left of the junction in $c$ that appears less well stained than its corresponding region in $d$ is out of focus in this image, giving a false impression of the lack of AChRs. In $b$, photographs of images obtained at 2 planes of focus have been combined for clarity.

Morphology of soleus NMJs does not change within 3 weeks

To determine whether the staining and viewing of NMJs triggered any morphological changes, I examined the arrangement of AChRs at superficial NMJs restained at relatively short times ( $<1$ month) after an initial viewing. Over the entire study, the frequency of successful relocation of NMJs at subsequent viewings was $96 \%$. In 83 junctions studied in animals between 7 and $24 \mathrm{~d}$ after staining and viewing with the lower toxin concentrations and illumination levels described in Materials and Methods, no changes were seen that could not be accounted for simply by the overall expansion of motor terminals, by distortion of the muscle caused by stretching it differently, or by viewing the muscle from a slightly different angle (Fig. 2). In 3 muscles from this group, motor nerve terminals were stained with TNBT immediately after the final viewing. At the 21 NMJs studied in these muscles, the shape of the nerve terminal revealed by TNBT staining matched the arrangement of fluorescently labeled AChRs (Fig. $2 c-e$ ). This showed that no covert alterations had occurred at nerve terminals that had not yet influenced the distribution of AChRs. Thus, at the concentrations of R- $\alpha$ BTX and illumination levels I employed, staining and viewing NMJs did not induce any changes in the morphology of junctions within 3 weeks.

\section{Morphology of soleus NMJs can change significantly over 3-6} months

In another group of animals, I viewed superficial NMJs with R- $\alpha$ BTX on 2 occasions separated by $3-6$ months. The majority of junctions appeared virtually identical at the second viewing (e.g., Fig. 3). Many junctions had expanded proportionately throughout their arbor (see, for example, Fig. 8), while others 
Figure 3. An NMJ stained with $\mathrm{R}-\alpha \mathrm{BTX}$ and studied in vivo on 2 occasions, about 5 months apart. The shape of this NMJ, as revealed by R- $\alpha$ BTX binding, was unchanged 155 $\mathrm{d}$ after the initial viewing.
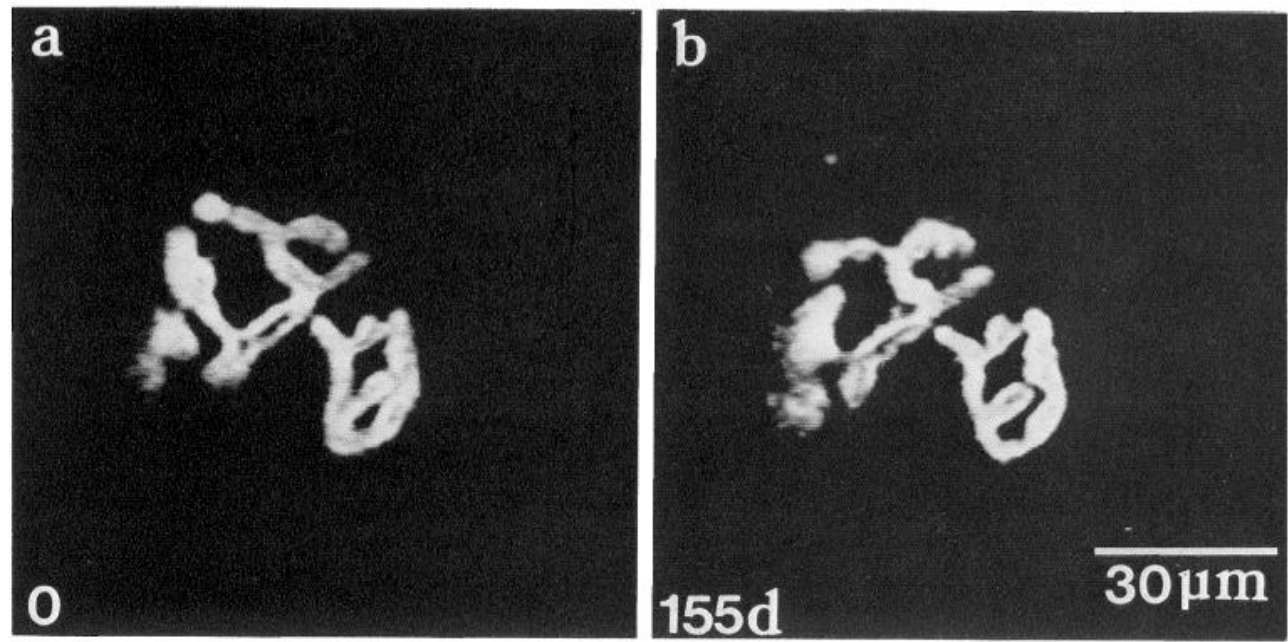

were distorted slightly but were nevertheless easily recognizable. These changes were discounted in the analysis; overall changes in the size of an NMJ were not quantified unless parts of its structure grew much more than others. A substantial fraction of junctions ( $\sim 25 \%$ on average), showed clear signs of such remodeling (Fig. 4). Some NMJs (15\%) lost part of their initial configuration (a deletion). A few junctions (9\%) developed entirely new branches (additions) or sometimes extended existing branches disproportionately: about $25 \%$ of the NMJs with clear changes showed both an addition and a deletion (Figs. 4, 5). The size distribution of changes that involved $5 \mu \mathrm{m}$ or or more of the AChR staining pattern is shown in Figure 6. This conservative criterion was chosen to ensure that only substantial changes were considered; the same criterion applies to all subsequent analyses. Overall, almost twice as many NMJs exhibited deletions as exhibited additions.

\section{Extent of remodeling depends on the time between the first and final viewings}

The animals were divided into 2 different groups on the basis of the time elapsed between the first and final viewings. The frequency of changes observed was greater after 6 months than after only 3 months (Table 1). In 10 animals reexamined at 3 months after the initial viewing, 9 of 78 NMJs (12\%) exhibited changes of $5 \mu \mathrm{m}$ or more, whereas 22 of $50(44 \%)$ NMJs changed in 9 animals examined 6 months after the initial viewing. These changes were not confined to a few, aberrant, NMJs in a few muscles: 5 of 10 animals reexamined after 3 months, and all 9 animals reexamined after 6 months, showed some remodeling. Furthermore, the frequency of remodeling was fairly consistent from animal to animal, although the small sample size in each animal precluded a meaningful analysis; the fraction of NMJs exhibiting remodeling varied from 0 to $33 \%$ in the 3 month group and from 33 to $66 \%$ in the 6 month group. The number of individual changes per NMJ also increased with time between the first and final viewings (Fig. 6) from a mean value of 0.16 \pm 0.06 (mean $\pm \mathrm{SE}$ ) for the 3 month group to $0.58 \pm 0.11$ for the 6 month group ( $p<0.005$, Student's $t$ test). There was no correlation between the frequency of remodeling and age or weight of the animals at the initial viewing or with weight gained during the course of the experiment. Thus, the frequency of changes seemed to depend on the total time between the first and final viewings. This further argues against the possibility that the changes observed were caused by the imaging procedure since it is unlikely that changes would continue to appear in response to a perturbation 3-6 months previously.

\section{NMJs studied on 3 separate occasions undergo no more remodeling than those studied only twice}

A possible side effect of the in vivo imaging approach might have been the induction of morphological changes that appeared very slowly. I therefore studied another group of animals in a slightly
Figure 4. An NMJ stained with $\mathrm{R}-\alpha \mathrm{BTX}$ and studied twice in vivo, 131 $\mathrm{d}$ apart. In $a$, the smaller arrowhead at the upper left indicates a $20 \mu \mathrm{m}$ length of R- $\alpha$ BTX staining clearly visible at the initial viewing that was absent 131 $\mathrm{d}$ later $(b)$. The larger arrowhead in $a$ points to a branch that subsequently grew much more than its parallel neighbor (compare $a$ and $b$ ). The fork at the bottom right in $b$ was also evident at day 0 but is not in focus in $a$. In $a$ and $b, 2$ different focal planes have been combined for clarity.
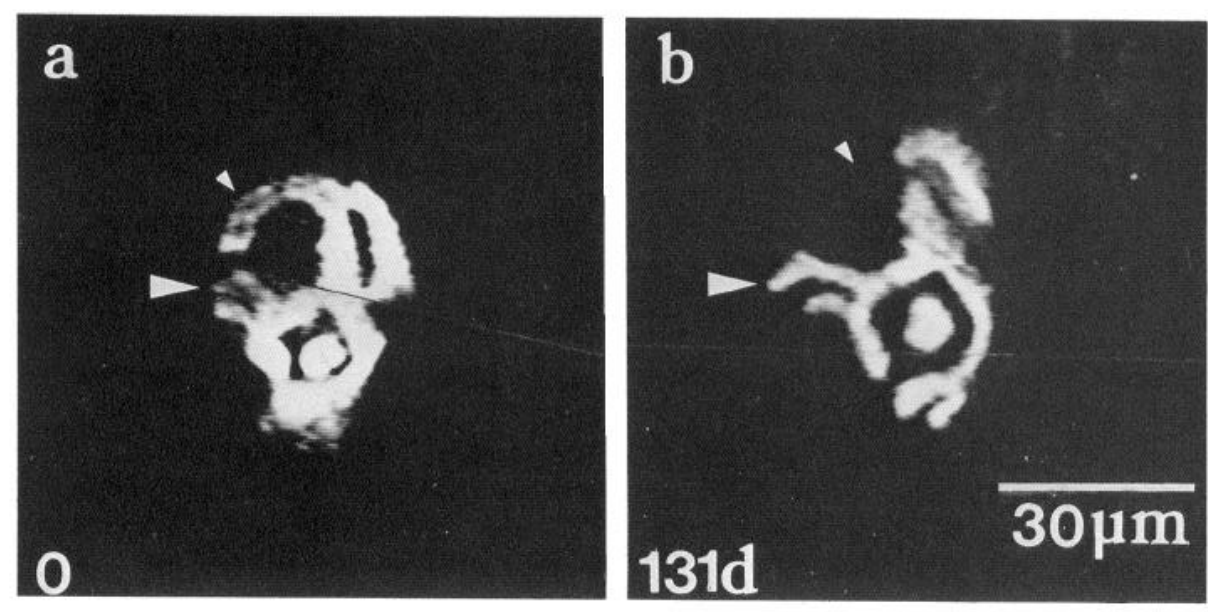

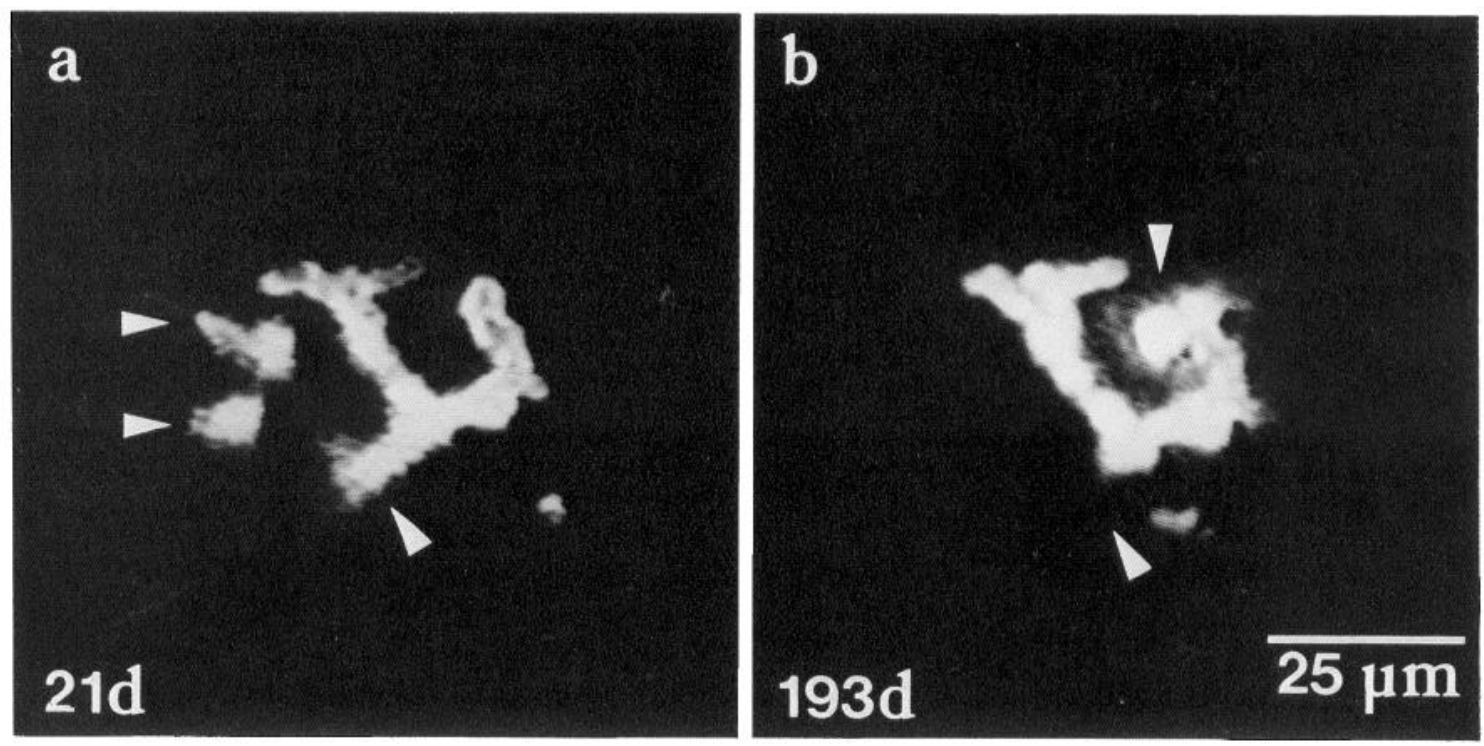

Figure 5. Two views of a junction studied 3 times, which underwent 3 separate deletions and an addition. The initial view at day 0 was identical to the second obtained at $21 \mathrm{~d}$ and is not shown here. Two large isolated regions of R- $\alpha \mathrm{BTX}$ staining at the left in $a$ were not present after restaining at $193 \mathrm{~d}(b)$. The tail of the Y-shaped configuration in $a$ is absent in $b$. A new region of R- $\alpha \mathrm{BTX}$ labeling appeared in $b$, indicated by the $u p p e r$ arrowhead.

different way: an initial viewing, a second viewing $7-21 \mathrm{~d}$ later, and a final viewing after 3-6 months (e.g., Fig. 7). Many of these animals provided data for the short-term experiments described above. The rationale for including an intermediate viewing step was that if staining and viewing triggered morphological changes (that appeared only after a delay), then more remodeling should be observed in NMJs studied 3 times than in those studied only twice. The frequency of remodeling was not altered by the addition of the extra viewing session (Table 2), arguing that the technique of in vivo imaging, if used carefully, does not by itself cause rearrangements of NMJs.

\section{Nerve terminal staining with TNBT}

Since R- $\alpha$ BTX binding delineates the location of AChRs, it is only an indirect indication of the shape of the overlying nerve terminal. To determine whether changes in the pattern of AChRs reflected alterations in the configuration of the presynaptic nerve terminal itself, I removed the soleus muscle after the final in vivo viewing of labeled AChRs and stained its motor nerve

\begin{tabular}{|c|c|c|}
\hline Parameter & 3 months & 6 months \\
\hline Number of animals & 10 & 9 \\
\hline Number of NMJs studied & 78 & 50 \\
\hline Percentage of NMJs changed ${ }^{a}$ & 12 & 44 \\
\hline Changes per $\mathrm{NMJ}^{b}$ & $0.16 \pm 0.06$ & $0.58 \pm 0.11$ \\
\hline
\end{tabular}

a Regions of an NMJ that appeared de novo, grew disproportionately to the rest of the junction, or disappeared completely were measured. Only changes equaling or exceeding $5 \mu \mathrm{m}$ in length were considered.

${ }^{b}$ Mean \pm SE. Calculated by dividing the number of changes observed in a particular muscle by the number of NMJs studied in that muscle and then averaging these values for each group.
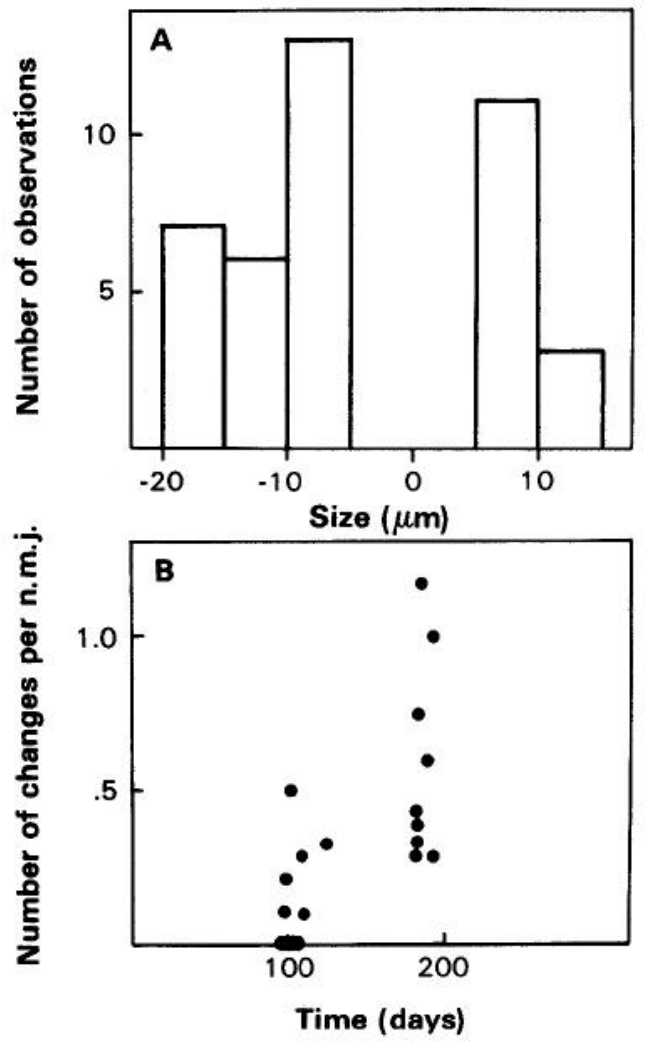

Figure 6. Quantitative analysis of morphological changes observed at soleus NMJs. $A$, Frequency distribution of the size of individual changes observed. Negative values represent deletions; positive values, additions. Not included are changes due to proportional growth of all branches of individual NMJs or apparent changes that were less than $5 \mu \mathrm{m}$. $B$, Number of changes observed in each muscle, divided by the number of NMJs studied in that muscle, plotted against the time elapsed between the first and final viewings. Difference between the means of the 3 and 6 month groups was significant ( $p<0.005$, Student's $t$ test). 
Figure 7. A single NMJ studied with $\mathrm{R}-\alpha \mathrm{BTX}$ in vivo on 3 occasions. $a$, Initial viewing; $b$, intermediate viewing after $14 \mathrm{~d}$; $c$, final viewing (110 d). A region that was well labeled at the first and second viewings (indicated by an arrow in $a$ and $b$ ) was stained only very faintly in $c . d$, Camera lucida drawing of this NMJ stained with TNBT immediately after the final in vivo viewing; ChE staining was not done. The region where AChRs seem to have been lost in $c$ stained clearly with TNBT in $d$ (arrow). This region was identified as the nerve entry to this NMJ by the presence of a myelin sheath around the (unstained) axon proximal to this region. In $a$ and $b, 2$ different focal planes have been combined.
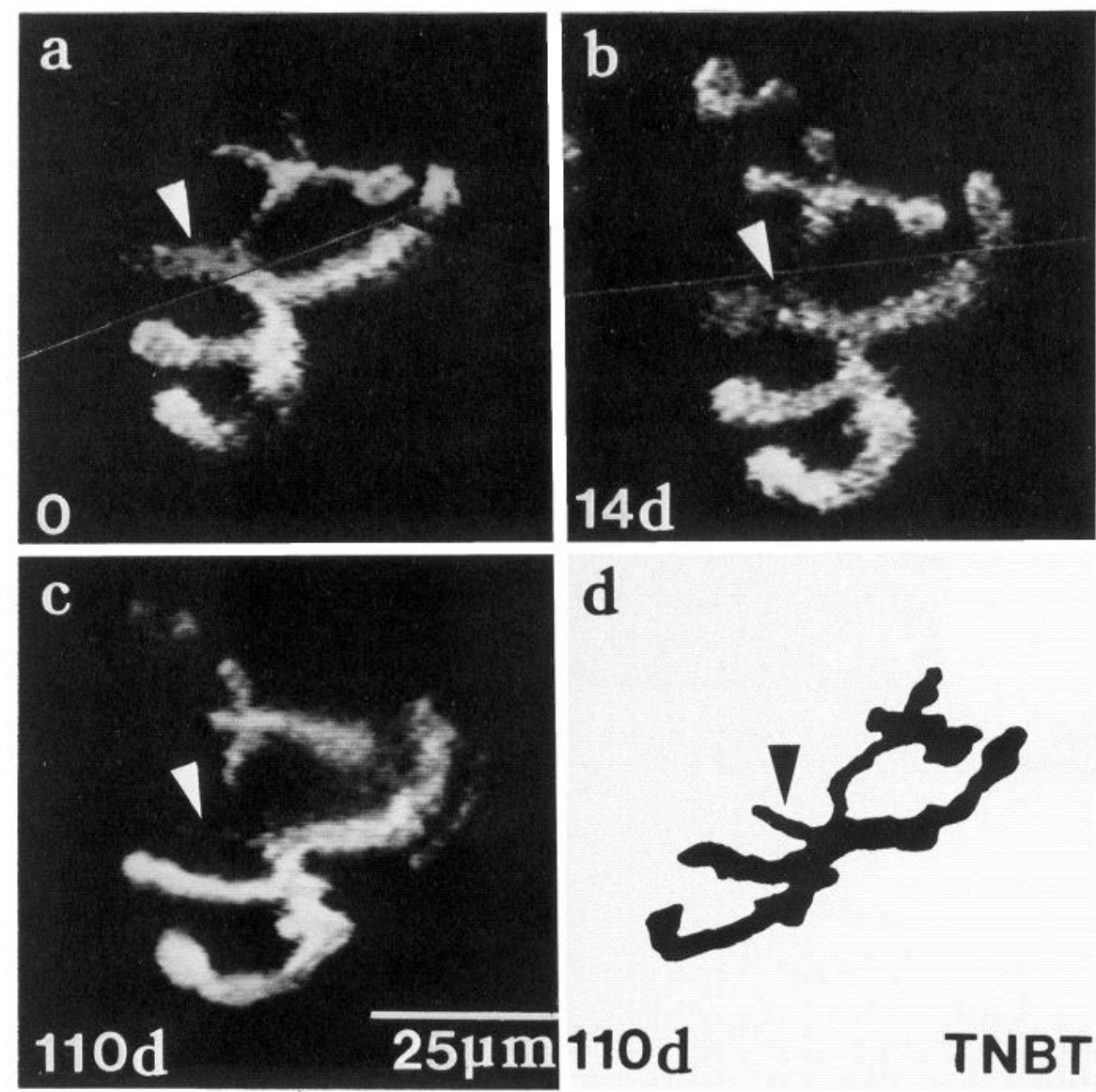

$110 d$ $25 \mu \mathrm{m} 110 \mathrm{~d}$

TNBT terminals with TNBT (Letinsky, 1983). In most experiments I also stained junctional ChE. In all examples of the de novo appearance of R- $\alpha \mathrm{BTX}$ staining (additions), I observed corresponding nerve terminal staining. In most instances in which a region of a NMJ appeared to have lost AChRs (deletions), there were no indications of nerve terminal staining. On the other hand, ChE could sometimes be detected at the site of a deletion (Fig. 8). Similar disparities between $\mathrm{ChE}$ staining and $\mathrm{AChR}$ distribution have been observed in adult frog muscle (Anzil et al., 1984). In all likelihood these instances represent recent deletions, since junctional ChE persists for some time after elimination of the presynaptic terminal. In some NMJs, at sites that had previously stained clearly with R- $\alpha \mathrm{BTX}$, neither AChRs nor AChE could be detected even though TNBT staining was present (see, for example, Fig. 7). This type of rearrangement always involved a region of the NMJ immediately distal to the termination of the myelin sheath. This suggests inat the relationship between the nerve terminal and its postsynaptic specializations can change, such that part of the NMJ, always its most proximal part, can no longer maintain postsynaptic specializations. Unfortunately, it is not clear exactly how many of the apparent deletions involved this kind of postsynaptic remodeling since TNBT staining was not always successful: 13 of the 40 changes I observed could not be verified with TNBT either because the staining was not done or was unsuccessful or because particular junctions could not be relocated after TNBT staining and mounting. Nevertheless, since $30 \%(8 / 27)$ of the changes at NMJs studied with TNBT clearly were of this postsynaptic type, and 6 of these were the sole change at that particular NMJ, these observations suggest that presynaptic ter- minal remodeling occurs in slightly fewer NMJs than suggested by R- $\alpha$ BTX binding. Nevertheless, at most NMJs, R- $\alpha$ BTX alone provides an accurate reflection of changes in the configuration of the presynaptic terminal.

\section{Discussion}

My observations indicate that $44 \%$ of NMJs in adult mouse soleus changed their shape noticeably over 6 months. The most common of these changes were deletions of individual branches or parts of branches. The addition of new branches, or the disproportionate elongation of an individual branch, occurred less frequently. Many NMJs expanded over the course of the study, not surprisingly since the animals gained weight $(\sim 10 \%)$ during this period. This overall enlargement of NMJs was not quantified since it is probably related simply to growth of the animal and, in particular, the soleus muscle. NMJs in adult mouse soleus muscle therefore appeared to undergo a significant degree of remodeling aside from growth, although substantially less than suggested by earlier studies (Cardasis and Padykula, 1981; Wernig et al., 1984; see below).

I was unable to obtain consistently clear staining of soleus motor nerve terminals with the mitochondrial-staining vital dyes $\mathrm{DiOC}_{2}$ or 4-Di-2-ASP (Yoshikami and Okun, 1984; Magrassi et al., 1987), primarily because of high background staining, even though I found the dyes to work excellently in a neighboring muscle (lateral gastrocnemius). This may be a consequence of the high mitochondrial content of slow-twitch muscles $(\mathrm{Pa}-$ dykula and Gauthier, 1970). I therefore employed a less direct indicator of nerve terminal configuration, the position of $\mathrm{AChRs}$ underlying the terminal. In mammalian skeletal muscles, AChRs 
Table 2. Frequency of remodeling at mouse soleus NMJs: comparison of NMJs studied twice with those studied 3 times

\begin{tabular}{llc} 
Parameter & 2 views & 3 views \\
\hline Number of animals & 10 & 9 \\
Number of NMJs studied & 66 & 62 \\
Percentage of NMJs changed $^{a}$ & 24 & 23 \\
Changes per NMJ $^{b}$ & $0.39 \pm 0.11$ & $0.32 \pm 0.13$
\end{tabular}

${ }^{a}$ Includes only changes of $\geqq 5 \mu \mathrm{m}$, as in Table 1 .

${ }^{b}$ Calculated as in Table 1 .

are clustered directly beneath motor nerve terminals and faithfully delineate the arrangement of the nerve terminal at the NMJ (see Fig. 2). Alterations in the shape of the nerve terminals, such as occur during terminal sprouting, cause a rapid redistribution of AChRs (Yee and Pestronk, 1987) so that this correspondence is maintained. In many experiments I stained nerve terminals nonvitally (TNBT) after the final viewing. With few exceptions, the arrangement of the nerve terminal revealed by TNBT staining agreed well with the AChR distribution revealed by R- $\alpha$ BTX binding in vivo. The exceptions were instances in which there appeared to be a change in the correspondence between the preand postsynaptic specializations, leaving a region of the presynaptic axon immediately adjacent to the terminal segment of the myelin sheath unapposed by postsynaptic AChRs or AChE. Thus, some instances of NMJ remodeling do not involve any obvious change in the arrangement of the presynaptic terminal. The observation of occasional postsynaptic remodeling in the absence of any obvious presynaptic change underscores the importance of using both pre- and postsynaptic probes to study synaptic remodeling. By my estimation, however, this class of remodeling can account for no more than $30 \%$ of the changes observed.

A puzzling feature of my results is that they seem to be inconsistent with studies suggesting that NMJs increase in morphological complexity with age (Tuffery, 1971; Tweedle and Stephens, 1981; Fahim et al., 1983; Wernig et al., 1984). In a pertinent study of mouse soleus (Wernig et al., 1984), the number of branch points of motor nerve terminals was found to increase between the ages of 3 and 6 months, a period also covered by my study. On the contrary, however, since I observed deletions more frequently than additions, my results suggest that soleus NMJs should become less complex with age. One possible explanation for this apparent discrepancy is that it might arise from the use of animals of a different strain and sex. Another possibility is that the difference might arise from a sampling bias in my experiments: I selected large, complex, and usually superficial NMJs for my study since these were the ones I could visualize with the greatest clarity. It might be that these NMJs were more likely to undergo deletions than additions. Indeed, Lichtman et al. (1987b) have reported that small NMJs tend to grow more than larger NMJs at any particular age. On the other hand, in at least one other study of adult mouse muscle, NMJs were found to decrease in complexity over a period corresponding to that of my experiments (Hopkins et al., 1985). Thus, while my results may be at odds with some studies, they are in accord with others. It is difficult to rule out the possibility that the repeated in vivo observations inhibited a normal increase in complexity or that some of the changes I observed were artifactual. While control experiments gave no indication that the staining and viewing procedure enhanced remodeling, it is hard-
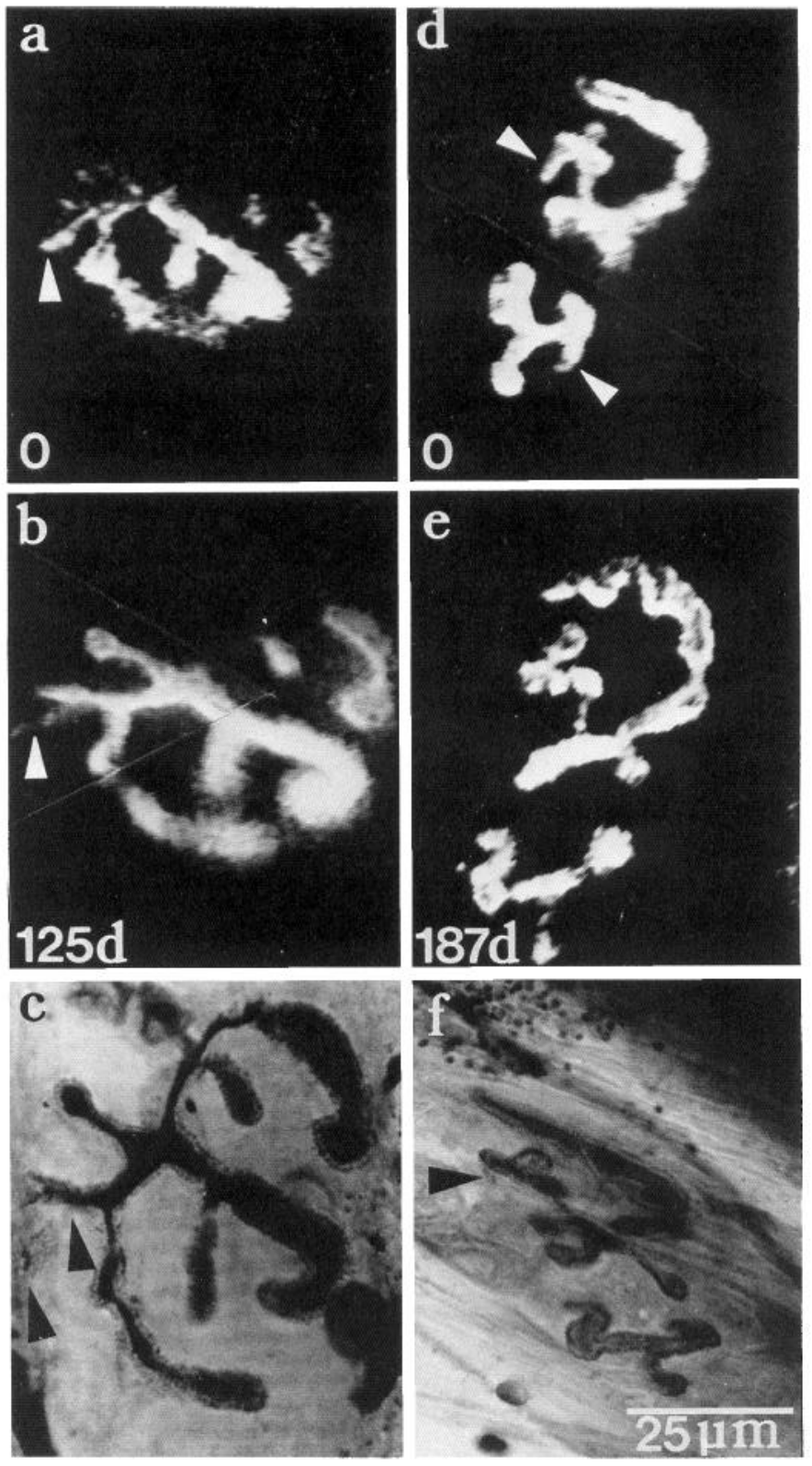

Figure 8. Two NMJs studied twice with several months between the initial and final viewings. $a$ and $b$, Views of the same junction stained with R- $\alpha \mathrm{BTX}$ in vivo, on 2 occasions $125 \mathrm{~d}$ apart. Part of the initial staining pattern in $a$ is virtually absent in $b$ (arrow). $c$, This region stained for ChE (black arrows). A myelinated axon can be seen entering this junction from the upper left; adjacent to this, a short region of unmyelinated axon that stains intensely with TNBT lacks corresponding ChE reaction product or $\operatorname{AChRs}(a, b) . d$ and $e$, Another junction stained and viewed twice in vivo, about 6 months apart. Two regions indicated with arrows in $d$ lacked R- $\alpha \mathrm{BTX}$ labeling in $e$; both of these failed to stain with TNBT in $f$, but one of them stained for ChE (black arrow). In $b$ and $d$, photographs obtained at 2 different focal planes have been combined for clarity.

er to address the question of inhibition. Indeed, the observation that remodeling frequency increased with time after the initial viewing is consistent with the decay of an inhibitory effect. In other systems, however, substantial remodeling has been observed using similar techniques to mine, even over relatively short intervals (Herrera and Banner, 1987; Purves et al., 1987). Furthermore, most NMJs in my own experiments grew over 
the course of the study, showing that growth of nerve terminals was certainly not prevented by the imaging technique.

The extent of remodeling I observed was substantially less than would be predicted from earlier studies of soleus NMJs using conventional histological and ultrastructural methods, especially considering the prevalence of small changes in the present study. In rat soleus between 3 and 5 months of age, Cardasis and Padykula (1981) found striking ultrastructural indications of reorganization in about $30 \%$ of NMJs at any one time. In a study of mouse soleus, Wernig et al. (1984) estimated that between 3 and 6 months of age each NMJ would undergo the loss or addition of at least one branch every $10 \mathrm{~d}$ or so. A similar difference between the degree of ongoing remodeling estimated by static and dynamic observations also exists for frog muscle (Wernig et al., 1980; Herrera and Banner, 1987; Herrera et al., 1988). Nevertheless, the frequency of remodeling I detected in soleus was much greater than that reported in a recent study of mouse sternomastoid by Lichtman et al. (1987a), also using direct observations in vivo. This difference might be explained by the different staining techniques employed: Lichtman et al. (1987a) used 4-Di-2-ASP, which stains nerve terminals somewhat incompletely, and small changes such as many of the ones I observed might have been missed or discounted. It is also possible, however, that the difference in the degree of NMJ remodeling between sternomastoid and soleus muscles might be related to one of several physiological or functional differences. For example, NMJs in fast-twitch muscles are generally of more complex morphology than in slow-twitch muscles (see, for example, Fahim et al., 1984; Lømo and Waerhaug, 1985). It may be, therefore, that some or all of the remodeling I observed, consisting mostly of branch deletions, is a consequence of the conversion of some soleus motor units from fast- to slowtwitch properties, as is known to occur in rat soleus during the first year of life (Kugelberg, 1976). The exact time during which this conversion occurs in mice, if at all, is not known. Another difference between soleus and sternomastoid NMJs that may be relevant to remodeling is the extent of their polyneuronal innervation at birth. Fast-twitch muscle fibers are generally contacted by fewer presynaptic axons at birth than fibers in the slow-twitch soleus (Brown et al., 1976; Van Essen, 1982). Thus, soleus NMJs probably undergo more extensive synapse elimination postnatally than NMJs in sternomastoid, although this process is probably completed long before the time at which I began my studies ( $\sim 3$ months). An intriguing alternative is that the difference in the degree of remodeling between soleus and sternomastoid might reflect different patterns or amounts of neural activity experienced by the 2 muscles. It might be possible to test this idea by varying motoneuronal activity while observing the morphology of individual NMJs in vivo.

\section{References}

Anzil, A. P., A. Bieser, and A. Wernig (1984) Light and electron microscopic identification of nerve terminal sprouting and retraction in normal adult frog muscle. J. Physiol. (Lond.) 350: 393-399.

Bailey, C. H., and M. Chen (1983) Morphological basis of long-term habituation and sensitization in Aplysia. Science 220:91-93.

Bailey, C. H., and M. Chen (1988) Long-term memory in Aplysia modulates the total number of varicosities of single identified sensory neurons. Proc. Natl. Acad. Sci. USA 85: 2373-2377.

Barker, D., and M. C. Ip (1966) Sprouting and degeneration of mammalian motor axons in normal and de-afferentated skeletal muscle. Proc. R. Soc. London 163: 538-554.
Brown, M. C., J. K. S. Jansen, and D. Van Essen (1976) Polyneuronal innervation of skeletal muscle in new-born rats and its elimination during maturation. J. Physiol. (Lond.) 261: 387-422.

Cardasis, C. A., and H. A. Padykula (1981) Ultrastructural evidence indicating reorganization at the neuromuscular junction in the rat soleus muscle. Anat. Rec. 200: 41-59.

Fahim, M. A., J. A. Holley, and N. Robbins (1983) Scanning and light microscopic study of age changes at a neuromuscular junction in the mouse. J. Neurocytol. 12:13-25.

Fahim, M. A., J. A. Holley, and N. Robbins (1984) Topographic comparison of neuromuscular junctions in mouse slow and fast twitch muscles. Neuroscience 13: 227-235.

Herrera, A. A., and L. R. Banner (1987) Direct observation of motor nerve terminal remodeling in living frogs. Soc. Neurosci. Abstr. 13: 1665.

Herrera, A. A., L. R. Banner, and N. Nagaya (1988) Discrepancies between histological and in vivo observations of motor nerve terminal remodelling. Soc. Neurosci. Abstr. 14: 1209.

Holland, R. L., and M. C. Brown (1980) Postsynaptic transmission block can cause terminal sprouting of a motor nerve. Science 207 : 649-651.

Hopkins, W. G., M. C. Brown, and R. J. Keynes (1985) Postnatal growth of motor nerve terminals in muscles of the mouse. J. Neurocytol. 14: 525-540.

Jordan, C. L., M. S. Letinsky, and A. P. Arnold (1988) Synapse elimination occurs late in the hormone-sensitive levator ani muscle of the rat. J. Neurobiol. 19: 335-356.

Karnovsky, M. J. (1964) The localization of cholinesterase activity in rat cardiac muscle by electron microscopy. J. Cell Biol. 23: 217-232.

Kelly, S. S., N. Anis, and N. Robbins (1985) Fluorescent staining of living mouse neuromuscular junctions. Pfluegers Arch. 404: 97-99.

Ko, C.-P. (1987) A lcctin, pcanut agglutinin, as a probc for the cxtracellular matrix in living neuromuscular junctions. J. Neurocytol. 16: 567-576.

Kugelberg, E. (1976) Adaptive transformation of rat soleus motor units during growth. J. Neurol. Sci. 27: 269-289.

Letinsky, M. S. (1983) Staining normal and experimental motor nerve terminals with tetrazolium salts. Stain Technol. 58: 21-27.

Lichtman, J. W., L. Magrassi, and D. Purves (1987a) Visualization of neuromuscular junctions over periods of several months in living mice. J. Neurosci. 7: 1215-1222.

Lichtman, J. W., M. M. Rich, and R. S. Wilkinson (1987b) The development and maintenance of synaptic connections at the neuromuscular junction. In From Message to Mind, pp. 168-179, Sinauer, Sunderland, MA.

Lnenicka, G. A., H. L. Atwood, and L. Marin (1986) Morphological transformation of synaptic terminals of a phasic motoneuron by longterm tonic stimulation. J. Neurosci. 6: 2252-2258.

Lømo, T., and O. Waerhaug (1985) Motor endplates in fast and slow muscles of the rat: What determines their differences? J. Physiol. (Paris) 80: 290-297.

Magrassi, L., D. Purves, and J. W. Lichtman (1987) Fluorescent probes that stain living nerve terminals. J. Neurosci. 7: 1207-1214.

Padykula, H. A., and G. F. Gauthier (1970) The ultrastructure of the neuromuscular junctions of mammalian red, white, and intermediate skeletal muscle fibers. J. Cell Biol. 466: 27-41.

Purves, D., and J. T. Voyvodic (1987) Imaging mammalian nerve cells and their connections over time in living animals. Trends Neurosci. 10: 398-404.

Purves, D., R. D. Hadley, and J. T. Voyvodic (1986) Dynamic changes in the dendritic geometry of individual neurons visualized over periods of up to three months in the superior cervical ganglion of living mice. J. Neurosci. 6: 1051-1060.

Purves, D., J. T. Voyvodic, L. Magrassi, and H. Yawo (1987) Nerve terminal remodeling visualized in living mice by repeated examination of the same neuron. Science 238: 1122-1126.

Robbins, N., and J. Polak (1987) Forms of growth and retraction at mouse neuromuscular junctions revealed by a new nerve terminal stain and correlative electron microscopy. Soc. Neurosci. Abstr. 13: 1007.

Rochel, S., and N. Robbins (1987) Acetylcholine receptor availability and transmission efficacy. Brain Res. 435: 41-47.

Rotshenker, S., and M. Tal (1985) The transneuronal induction of sprouting and synapse formation in intact mouse muscles. J. Physiol. (Lond.) 360: 387-396. 
Sanes, J. K., and J. M. Cheney (1982) Lectin binding reveals a synapsespecific carbohydrate in skeletal muscle. Nature 300:646-647.

Scott, L. J. C., F. Bacou, and J. R. Sanes (1988) A synapse-specific carbohydrate at the neuromuscular junction: Association with both acctylcholinesterase and a glycolipid. J. Neurosci. 8: 932-944.

Sotelo, C., and S. L. Palay (1971) Altered axons and axon terminals in the lateral vestibular nucleus of the rat. Lab. Invest. 25: 653-671.

Tuffery, A. R. (1971) Growth and degeneration of motor end-plates in normal cat hind limb muscles. J. Anat. 110:221-247.

Tweedle, C. D., and K. E. Stephens (1981) Development of complexity in motor nerve endings at the rat neuromuscular junction. Neuroscience $6: 1657-1662$.

Van Essen, D. C. (1982) Neuromuscular synapse elimination. In Neuronal Development, N. C. Spitzer, ed., pp. 333-376, Plenum, New York.

Wernig, A., M. Pécot-Dechavassine, and H. Stöver (1980) Sprouting and regression of the nerve at the frog neuromuscular junction in normal conditions and after prolonged paralysis with curare. J. Neurocytol. 9: 277-303.

Wernig, A., J. J. Carmody, A. P. Anzil, E. Hansert, M. Marciniak, and H. Zucker (1984) Persistence of nerve sprouting with features of synapse remodelling in soleus muscles of adult mice. Neuroscience II: $241-253$

Wigston, D. J. (1987) Repeated imaging of neuromuscular junctions in mouse soleus muscles in vivo. Soc. Neurosci. Abstr, 13: 1007.

Yee, W. C., and A. Pestronk (1987) Mechanisms of postsynaptic plasticity: Remodeling of the junctional acetylcholine receptor cluster induced by motor nerve terminal outgrowth. J. Neurosci. 7: 20192024.

Yoshikami, D., and L. M. Okun (1984) Staining of living presynaptic nerve terminals with selective fluorescent dyes. Nature 310:53-56. 